\title{
High prevalence of extended-spectrum beta-lactamase-producing Klebsiella pneumoniae in a tertiary care hospital in Tehran, Iran
}

\author{
Hadi Mehrgan ${ }^{1,2}$, Mohammad Rahbar ${ }^{3}$ and Zohreh Arab-Halvaii ${ }^{1}$ \\ ${ }^{1}$ Department of Pharmaceutics, School of Pharmacy, Shahid Beheshti University of Medical Sciences, Tehran, Iran \\ ${ }^{2}$ Pharmaceutical Sciences Research Center, Shahid Beheshti University of Medical Sciences, Tehran, Iran \\ ${ }^{3}$ Iranian Health Reference Laboratories, Ministry of Health, Tehran, Iran
}

\begin{abstract}
Background: Extended-spectrum beta $(\beta)$-lactamase (ESBL)-producing enterobacteria are major emerging pathogens in nosocomial infections.

Methodology: The combination disk synergy test was used to evaluate 202 consecutive non-repeated Klebsiella pneumoniae (K. pneumonia) strains for ESBL production. The strains were isolated from various clinical specimens of hospitalized patients over the period from July 2005 to March 2007. Their antibiotic susceptibility pattern was also determined by the disk diffusion method. Demographic and medical data of the patients were recorded using a questionnaire.

Results: One hundred and fifty-seven (77.7\%) of the isolates were confirmed as ESBL-producers. By univariate analysis, young age, stay in intensive care unit (ICU)/medical wards, recent stay in ICU, and number of days of ICU stay were found to be risk factors for acquisition of resistant bacteria ( $\chi 2$ and Mann-Whitney $\mathrm{U}$ tests, $\mathrm{P}<0.05)$. However, binary logistic multivariate regression analysis confirmed that stay in ICU [Odds ratio (OR) 6.09, 95\% confidence interval (CI) 2.36-15.72; P $<0.001$ ] or medical wards [OR 3.72, 95\% CI 1.42-9.75; P = 0.007] were significantly associated with ESBL production. Imipenem, ofloxacin, cefoxitin and norfloxacin (against urinary isolates) were found to be highly active against ESBL-producing isolates in vitro (100\%, 75.2\%, 69.4\% and 66.7\% susceptibility, respectively). In addition to most $\beta$-lactams, they showed co-resistance with other antibiotics such as ciprofloxacin, aminoglycosides, trimethoprim/sulfamethoxazole and tetracycline.

Conclusion: Our results showed a high prevalence of ESBL-producing K. pneumoniae in our hospital setting. As the available treatment options are limited, antibiotic control policies together with the implementation of infection control measures remain of high importance.
\end{abstract}

Key words: Klebsiella pneumoniae, ESBL, beta-lactamases, nosocomial infection, Enterobacteriaceae

J Infect Dev Ctries 2010; 4(3):132-138.

(Received 09 September 2009 - Accepted 29 December 2009)

Copyright $(92010$ Mehrgan et al. This is an open-access article distributed under the Creative Commons Attribution License, which permits unrestricted use, distribution, and reproduction in any medium, provided the original work is properly cited.

\section{Introduction}

Extended-spectrum beta $(\beta)$-lactamases (ESBLs) are enzymes conferring broad resistance to penicillins, cephalosporin and monobactams, but not carbapenems. ESBLs are often plasmid-mediated and most are members of the TEM and SHV families of enzymes. Plasmids containing genes encoding for ESBLs often contain resistance determinants for other classes of antimicrobial agents and are readily transmissible from strain to strain and between different species of enteric Gram-negative bacilli [1]. ESBLs are detected most commonly in Klebsiellae pneumonia (K. pneumoniae) and Escherichia coli (E. coli). ESBL-producing $K$. pneumoniae strains have been implicated in numerous outbreaks of nosocomial infections over the last two decades $[2,3]$.

Considering the extensive use of $\beta$-lactam antibiotics including third-generation cephalosporins for the treatment of both hospital- and communityacquired infections in Iran, prevalence of ESBL positive clinical isolates is very probable. A single report on these resistant bacteria from Iran denoted the occurrence of $K$. pneumoniae producing ESBL as high as $44.5 \%$ [4]. In this study, we determined the prevalence of ESBL production among $K$. pneumoniae isolated from inpatients, their susceptibility patterns, as well as the risk factors associated with the acquisition of these isolates.

\section{Materials and Methods}

Study population and specimen types

This study was conducted at Milad Hospital, a tertiary care hospital with 1,000 beds belonging to The Social Security Organization, located in Tehran, Iran. From July 2005 to March 2007, consecutive, non-duplicate nosocomial isolates of $K$. pneumoniae 
were collected from various specimens of patients who were hospitalized for at least 48 hours. Specimens included urine, wound, respiratory tube, blood, cerebrospinal fluid, eye swab, etc.

Medical and demographic data of the patients were collected using a questionnaire. Data recorded were as follows: demographic characteristics (age, gender); underlying diseases (diabetes mellitus, chronic renal failure, cancer, hepatitis and cardiac disorders); presence of intravascular or urinary catheters; hospitalization; history of intensive care unit (ICU) stay and length of stay; being on mechanical ventilation; previous therapy with thirdgeneration cephalosporins (ceftazidime, cefotaxime and ceftriaxone); recent hospitalization; and recent surgery.

Previous hospitalization was defined as admission to a hospital ward within one year prior to the current admission. Recent surgery was defined as any major surgical procedure performed in the operating room within the past year. Previous therapy with third-generation cephalosporins was defined as treatment with any of the antibiotics ceftazidime, cefotaxime and ceftriaxone given for at least two days within 60 days preceding isolation of the organism.

\section{Microbiological methods}

All samples were routinely cultured on MacConkey and blood agar plates. Blood and sputum were cultured on chocolate agar plates. All suspected colonies were identified by Gram staining, colony characteristics, motility and biochemical reactions [5].

\section{Antibiotic susceptibility testing}

Antimicrobial susceptibility testing of the isolated organisms was performed by the disk diffusion technique according to the recommendations of the Clinical and Laboratory Standards Institute (CLSI) [6]. Data were reported as sensitive, intermediate or resistant. For statistical purposes, in some cases the susceptibility data were categorized as susceptible and non-susceptible (including intermediate and resistant groups). All disks were obtained from Oxoid Ltd. (Basingstoke, UK). The antibiotic potency of the disks was standardized against the reference strains $E$. coli ATCC 25922, E. coli ATCC 35218 and Pseudomonas aeruginosa ATCC 27853.

Detection of ESBL
Production of ESBL in the isolates was determined by the combination disk technique using antibiotic disks containing ceftazidime $(30 \mu \mathrm{g})$, cefotaxime $(30 \mu \mathrm{g})$ and cefpodoxime $(10 \mu \mathrm{g})$ either alone or in combination with clavulanic acid $(10 \mu \mathrm{g})$. The results were interpreted according to the recommendations of the CLSI [6], providing the isolates were shown to be ESBL-producers with respect to the three antibiotics. K. pneumoniae ATCC 700603 and E. coli ATCC 25922 were used as positive and negative controls for ESBL production, respectively.

\section{Statistical analysis}

Data were entered into a database using SPSS 11.5 for Windows (SPSS Inc., Chicago, IL). In the case of bivariate comparisons, the Pearson $\chi 2$ test was used for categorical and Mann-Whitney U-test for quantitative variables. To analyze any association between the presence of ESBL-producing isolates and the possible covariates, a step-by-step analysis was designed. In the first step, using an appropriate preliminary analysis, factors highly unlikely to be correlated with the presence of ESBL were determined (covariates with P-values $>0.2$ were not included in the final analysis). In the second step, any interactions between eligible factors obtained in the first step were determined using the $\chi^{2}$ test, MannWhitney U-test or Kruskal-Wallis H-test. Those interactions with $\mathrm{P}$-values $<0.05$ were then considered for inclusion in the multivariate model. In the final step, a multivariate logistic regression analysis was performed using a backward stepwise selection process. A two-tailed $\mathrm{P}$-value $<0.05$ was considered statistically significant.

\section{Results}

During the study period, a total of $202 \mathrm{~K}$. pneumoniae were isolated from a range of clinical specimens of patients hospitalized in various wards of Milad Hospital, Tehran, Iran. K. pneumoniae isolates were frequently isolated from patients at the extreme ages (i.e., less than two years [35.6\%] and $61+$ years [30.7\%]; males $(57.0 \%)$; cases in ICU wards $(53.0 \%)$; those recently stayed in ICU (59.4\%); and those having intravascular or urinary catheters $(63.4 \%)$, often cultured from urine samples $(45.0 \%)$. As few as $0.99 \%$ of the patients had undergone renal transplant. Only one case had a history of stay in a long-term care facility.

The prevalence of ESBL-producing $K$. pneumoniae was $77.7 \%$ (157/202). As shown in 
Table 1, based on the Mann-Whitney $U$ and $\chi 2$ tests, factors such as young age, stay in ICU/medical wards, history of ICU stay and its length were found to be major risk factors for the acquisition of ESBLproducing $K$. pneumoniae $(\mathrm{P}<0.05)$. All $K$. pneumoniae isolated from blood or eye specimens were ESBL-producing. Moreover, production of ESBL in $K$. pneumoniae isolated from various specimens was analyzed using the $\chi^{2}$ test. $K$. pneumoniae isolated from urine samples was shown to produce ESBL at a significantly different rate $(\mathrm{P}<$ 0.05) depending on gender (data not shown). Specimens were put into two groups, urine and nonurine, and the $\chi 2$ test repeated. ESBL production with $84 \%$ vs. $60 \%$ was significantly higher $(\mathrm{P}=0.018)$ in $K$. pneumoniae isolated from males' urine samples. As shown in Table 1, prevalence of ESBL production was higher in $K$. pneumoniae isolated from non-urine samples. Patients were grouped as $\leq 18$ years old and $19+$ years old and their specimens were categorized as urine, respiratory tube and others, for statistical purposes. ESBL production was more often seen in $K$. pneumoniae isolated from respiratory tube specimens of patients 19+ years old. However, this difference was not significant. Conversely, the prevalence of ESBL production was much greater $(\mathrm{P}<0.05)$ in $K$. pneumoniae isolated from non-respiratory tube specimens of patients $\leq 18$ years old (data not shown). By multivariate analysis, $K$. pneumoniae isolation from specimens of patients staying either in ICU (odd ratio (OR) 6.09, 95\% confidence interval (CI): 2.36$15.72 ; \mathrm{P}<0.001)$ or in medical wards (OR $3.72,95 \%$ CI 1.42-9.75; $\mathrm{P}=0.007)$ remained significantly associated with ESBL production.

The results of antibiotic susceptibility testing are shown in Table 2. In general, susceptibility rates of $K$. pneumoniae isolates to third- and fourth-generation cephalosporins, aztreonam, amoxicillin/clavulanic acid, piperacillin/tazobactam, aminoglycosides and tetracycline were low. Fluoroquinolones, cefoxitin, trimethoprim/sulfametoxazole (SXT) and nitrofurantoin (against urinary isolates) showed moderate activity on $K$. pneumoniae isolates. Imipenem was shown to be the most active antibiotic against these isolates. ESBL production rendered $K$. pneumoniae isolates resistant to most $\beta$-lactams as well as to non- $\beta$-lactams such as ciprofloxacin and aminoglycosides. Their resistance rates to most antibiotics were significantly $(\mathrm{P}<0.05)$ higher than those of ESBL-negative isolates. However, all ESBL producers were susceptible to imipenem. Susceptibility rate was moderate to ofloxacin (75.2\%), norfloxacin (66.7\%) and cefoxitin (69.4\%). Aminoglycosides, with ca. $65 \%$ susceptibility, showed decreased activity even against ESBLnegative $K$. pneumoniae. ESBL production by the isolates could not markedly influence their susceptibility pattern to cefoxitin, ofloxacin, SXT and nitrofurantoin. Finally, tetracycline was shown to be of no use in the treatment of infections caused by $K$. pneumoniae.

\section{Discussion}

Although many international studies have addressed the emergence of ESBL-producing $K$. pneumoniae, there is only one report on this issue from Iran [4]. The current study demonstrated a very high prevalence of ESBL production $(77.7 \%)$ by $K$. pneumoniae isolates in our hospital setting. This high ESBL frequency may have been caused by the excessive use of broad-spectrum antibiotics in our hospital and to a higher level in our community setting, together with a lack of attention to laboratory screening of ESBL production by clinical isolates. Actually, ceftriaxone was the most common antibiotic in the hospital, as observed during this study and in our previous report [7], despite its reduced activity against pathogens such as $K$. pneumoniae (susceptibility 13.4\%). On the other hand, the high rate of ESBL production could possibly be due to the spread of one single clone and/or plasmid within our hospital setting. Owing to a number of limitations, we could not exclude this possibility by determining plasmid profiles and pulse-field gel electrophoresis patterns of the isolates. The prevalence of ESBL-producing $K$. pneumoniae has been reported at hospitals in the SENTRY surveillance programme [8]. K. pneumoniae isolates with an ESBL phenotype were more prevalent in Latin America (45.4\%), followed by the Western Pacific region $(24.6 \%)$, Europe $(22.6 \%)$, the United States $(7.6 \%)$ and Canada (4.9\%). In the Middle East, where Iran is located, prevalence of ESBL-producing K. pneumoniae is as follows: Saudi Arabia 12.2\% [9], Lebanon $20.0 \%$ [10], Egypt $37.5 \%$ [11], Israel $32.0 \%-79.0 \% \quad[12,13]$, Turkey $50.0 \%$ [14], and Jordan $80 \%$ [15]. In Pakistan, a study performed in Karachi showed an ESBL prevalence of $36.0 \%$ in nosocomial $K$. pneumoniae isolates [16]. A higher rate was reported from India where $80.0 \%$ of $K$. pneumoniae isolates in a tertiary care center were labeled as ESBL producers [17]. The ESBL prevalence in our study may be underestimated 
Table 1. Distribution of patients based on the studied variables and the results of univariate analyses $(n=202)$.

\begin{tabular}{|c|c|c|c|c|}
\hline Variable $^{a}$ & $\begin{array}{c}\text { ESBL-non- } \\
\text { producer }(n=45)\end{array}$ & $\begin{array}{l}\text { ESBL-producer } \\
\quad(n=157)\end{array}$ & $\begin{array}{c}\text { Risk ratio }(95 \% \\
\text { CI })\end{array}$ & $P$-value ${ }^{\mathrm{b}}$ \\
\hline Age (years) & $47.1 \pm 29.5$ & $31.0 \pm 31.8$ & - & 0.008 \\
\hline Hospital admission ward & & & - & $<0.001$ \\
\hline ICU & $16(15.1)$ & $90(84.9)$ & & \\
\hline Medical & $16(22.5)$ & $55(77.5)$ & & \\
\hline ICU stay & $18(15.0)$ & $102(85.0)$ & $2.78(1.41-5.49)$ & 0.003 \\
\hline Days of ICU stay & $9.5 \pm 11.5$ & $19.7 \pm 31.3$ & - & 0.032 \\
\hline Intravascular or urinary catheter & $27(21.1)$ & $101(78.9)$ & $1.20(0.61-2.37)$ & 0.603 \\
\hline Mechanical ventilation & $10(14.3)$ & $60(85.7)$ & $2.17(1.00-4.69)$ & 0.052 \\
\hline Surgery in the preceding year & $9(25.7)$ & $26(74.3)$ & $0.79(0.34-1.84)$ & 0.655 \\
\hline Hospitalization in the preceding year & $22(27.5)$ & $58(72.5)$ & $0.67(0.34-1.30)$ & 0.300 \\
\hline Urine & $25(27.5)$ & $66(72.5)$ & & \\
\hline Respiratory tube & $10(19.2)$ & $42(80.8)$ & & \\
\hline Others & $10(16.9)$ & $49(83.1)$ & & \\
\hline
\end{tabular}

ESBL, extended-spectrum $\beta$-lactamase; CI, confidence interval; ICU, Intensive Care Unit.

a Quantitative variables, i.e. age and length of ICU stay, are presented as mean value \pm standard deviation; categorical variables are presented as number $(\%)$.

b Univariate analysis was performed using Mann-Whitney U-test for quantitative and the $\chi^{2}$ test for categorical variables. A P-value $<0.05$ was considered statistically significant.

c Only 124 patients could provide an answer to this question and thus were entered in the statistical analysis.

Table 2. Antibiotic susceptibility rate $(\%)$ of extended-spectrum $\beta$-lactamase (ESBL)-positive and ESBL-negative Klebsiella pneumoniae isolates.

\begin{tabular}{|c|c|c|c|c|}
\hline Antibiotic & $\begin{array}{c}\text { ESBL-non- } \\
\text { producer }(n=45)\end{array}$ & $\begin{array}{c}\text { ESBL-producer } \\
(n=157)\end{array}$ & $\begin{array}{c}\text { Total } \\
(n=202)\end{array}$ & $P$-value ${ }^{\mathrm{a}}$ \\
\hline Cefotaxime & 57.8 & 0.6 & 13.4 & $<0.001$ \\
\hline Ceftriaxone & 57.8 & 0.6 & 13.4 & $<0.001$ \\
\hline Cefepime & 71.1 & 9.6 & 23.3 & $<0.001$ \\
\hline Aztreonam & 57.8 & 0.6 & 12.9 & $<0.001$ \\
\hline Imipenem & 93.3 & 100.0 & 98.5 & 0.01 \\
\hline Amoxicillin/clavulanic acid & 53.3 & 4.5 & 15.3 & $<0.001$ \\
\hline Piperacillin/tazobactam & 48.9 & 28.7 & 33.2 & 0.013 \\
\hline Ciprofloxacin & 71.1 & 47.8 & 53.0 & 0.007 \\
\hline Ofloxacin & 86.7 & 75.2 & 77.7 & 0.109 \\
\hline Norfloxacin $^{\text {b }}$ & 76.0 & 66.7 & 69.2 & 0.454 \\
\hline Tetracycline & 2.2 & 1.9 & 2.0 & 1.000 \\
\hline Nitrofurantoin ${ }^{\mathrm{b}}$ & 73.3 & 60.0 & 63.6 & 0.531 \\
\hline
\end{tabular}

SXT, trimethoprim/sulfamethoxazole

a Exact P-values were determined by the $\chi^{2}$ test. For statistical analysis, the response of isolates to antibiotics was categorized as susceptible and non-susceptible (consisting of intermediate and resistant groups). A P-value $<0.05$ was considered statistically significant.

b Norfloxacin and nitrofurantoin were respectively tested against 91 and 55 of urinary isolates only. 
owing to the limitations of phenotypic detection of ESBL-producing isolates $[18,19]$.

In terms of risk factors for the acquisition of ESBL-producing $K$. pneumoniae in this study setting, age was shown to be one of these factors. In fact, ESBL production was most frequent in patients at the lower extreme of age, who have underdeveloped immunity and are therefore more likely susceptible to infections with opportunistic pathogens such as $K$. pneumoniae. This finding was similar to that obtained by Jabeen et al. [16]. The increased ESBL prevalence in the lower age group studied herein is likely to be attributed to high ESBL load in our setting, a hypothesis supported by other surveillance studies from Asia and the Asian Pacific region [20]. ESBL-producing isolates from urine were more common in males than females, in agreement with a previous report [21]. ESBL-producing $K$. pneumoniae were frequently isolated from ICU patients, who tended to be more debilitated, more likely to need ventilatory assistance, and had greater exposure to antimicrobial agents. This risk factor has also been confirmed by other investigators [21]. Sixty-four out of 72 infants $(88.9 \%)$ were staying in neonatal ICUs with an ESBL-positivity rate of $89.1 \%$. Outbreaks of ESBL-producing $K$. pneumoniae in NICUs have been notable for high attack rates and the large number of colonized infants [22]. In contrast to the report by Graffunder et al. [21], previous use of third-generation cephalosporins played no role as risk factor for ESBL production in our study. This could be related to the overuse of antibiotics in Iran's community and hospital settings, resulting in selection and spread of resistant organisms. Other risk factors such as male gender, intravascular/urinary catheterization, mechanical ventilation, prior surgery/hospitalization, and underlying diseases were not shown to be significantly associated with the acquisition of ESBL-producing isolates, in contrast to previous reports [21,23], possibly due to a masking effect of high ESBL load in our setting.

The overall resistance rate of the $K$. pneumoniae isolates studies was distressingly high, at $86.6 \%$ for ceftriaxone, $66.8 \%$ for piperacillin/tazobactam, $47.0 \%$ for ciprofloxacin, $72.8 \%$ for amikacin, $78.2 \%$ for gentamicin, $49.5 \%$ for SXT, and as high as $98.0 \%$ for tetracycline (Table 2). ESBL-producing isolates in this study were found to be concomitantly resistant to various antibiotic classes, indicating the co-transfer of a range of genes accounting for resistance to these antibiotics [1]. Therefore, therapeutic choices became limited in our hospital. Based on our in vitro findings, imipenem was the most effective antibiotic against ESBL-producing $K$. pneumoniae (100\%), followed by ofloxacin (75.2\%) and cefoxitin (69.4\%). $K$. pneumoniae isolates, even those producing ESBL, were reported to be $100 \%$ sensitive to imipenem by different research groups, e.g., Jones et al. [24]. Many investigators prefer imipenem and meropenem as the drugs of choice for life-threatening infections due to ESBL-producing Enterobacteriaceae or in an outbreak setting [18]. However, to preserve the therapeutic value of carbapenems, based on institutional patterns of susceptibility results, piperacillin/tazobactam, fluoroquinolones or an aminoglycoside would be preferable. Ofloxacin, with $75.2 \%$ susceptibility, was shown to be moderately active against ESBL-producing isolates, in contrast to ciprofloxacin. The resistance to ciprofloxacin in this study was comparable to previous reports $[8,9]$ but was higher than that observed in other studies [14,24]. However, some other reports implied higher resistance to this antibiotic $[10,12,17]$. Reports have shown a close association between ESBL production and ciprofloxacin resistance [22]. Cefoxitin, which resists hydrolysis by ESBLs, showed only fair activity against isolates producing ESBL in our study. This could be attributable to the concomitant presence of a plasmid-mediated AmpC-type $\beta$ lactamase that effectively hydrolyses this antibiotic or to porin-deficient mutants [18]. Reduced in vitro susceptibility to aminoglycosides in the isolates (< $30 \%$ ) has voided their use in the treatment of infections. Similar findings have been documented previously $[10,17]$. Alternatively, some other studies have demonstrated better aminoglycosides activity against ESBL-producing $K$. pneumoniae [8,24]. Cefepime showed a very low activity (Table 2) against $K$. pneumoniae producing ESBL. Similar results were found in another study [9]. In contrast to our finding, this fourth-generation cephalosporin with a susceptibility rate of $>87 \%$ has been reported to be highly active against ESBL-producing $K$. pneumoniae in Canada and the USA [8]. Nevertheless, even if it shows such in vitro activity, cefepime would not be recommended for empirical therapy of Gram-negative bacillary infections in centers where ESBL-producing organisms predominate [18]. This is also true for other cephalosporins and penicillins to which all confirmed ESBL-producing strains are reported as resistant for clinical use [6]. One of the most striking findings of the present study was the high prevalence of resistant 
strains among ESBL-producing $K$. pneumoniae to piperacillin/tazobactam. Another study group from Iran reported $33 \%$ resistance to this antibiotic combination, which was much less than our finding [4]. This resistance rate, however, was consistent with those from Lebanon [10], Israel [12], and India [17]. In fact, although piperacillin/tazobactam has been used successfully to treat bloodstream infections in preterm infants and UTI caused by ESBLproducing $K$. pneumoniae [25], it should be noted that therapeutic failures with this antibiotic combination have already been documented [22] and thus in vitro susceptibility may not necessarily predict in vivo efficacy. With moderate activity against urinary ESBL-producing isolates, nitrofurantoin might be useful in the therapy of lower urinary tract infection, at least in patients who could take oral medications. Feizabadi et al. reported higher resistance (74\%) of ESBL-producing K. pneumoniae to this antibiotic [4]. With a susceptibility rate as low as $47 \%$, SXT was shown to be of no value in the treatment of infections caused by ESBL-producing isolates. This finding was much worse than that reported in previous studies [8], but comparable with some others $[4,23]$.

Briefly, a high prevalence of ESBL-producing $K$. pneumoniae was observed in our hospital setting. As the available treatment options are limited, antibiotic control policies together with the implementation of infection control measures remain of high importance. Because of the new challenges presented by the changing nature and distribution of these enzymes, clinicians need to be familiar with the clinical significance of these enzymes, and clinical microbiology laboratories require adopting a technique most appropriate to them for their detection. Conducting molecular and epidemiological studies will help to identify various types of ESBLs and establish the relationship between ESBL-producing isolates. A national surveillance study in Iran as a vast country is also recommended.

\section{Acknowledgments}

The authors cordially thank the chairman of Milad Hospital as well as their co-operative colleagues in the microbiology laboratory. This work was financially supported by grant No. 1025 from Shahid Beheshti University of Medical Sciences.

\section{References}

1. Paterson DL (2006) Resistance in Gram-negative bacteria: Enterobacteriaceae. Am J Med 119: S20-28.

2. Tzelepi E, Magana Ch, Platsouka E, Sofianu D, Paniara O, Legakis NJ et al. (2003) Extended-spectrum $\beta$-lactamase types in Klebsiella pneumoniae and Escherichia coli in two Greek hospitals. Int J Antimicrob Agents 21: 285-288.

3. Hirakata Y, Matsuda J, Miyazaki Y, Kamihira S, Kawakami $S$, Miyazawa $\mathrm{Y}$ et al. (2005) Regional variation in the prevalence of extended-spectrum $\beta$-lactamase-producing clinical isolates in the Asia-Pacific region (SENTRY 19982002). Diagn Microbiol Infect Dis 52: 323-329.

4. Feizabadi MM, Etemadi G, Yadegarinia D, Rahmati M, Shabanpoor S, Bokaei S (2006) Antibiotic-resistance patterns and frequency of extended-spectrum $\beta$-lactamaseproducing isolates of Klebsiella pneumoniae in Tehran. Med Sci Monit 12: BR362-365.

5. Forbes BA, Sahm DF and Weissfeld AS (2002) Bailey and Scott's Diagnostic Microbiology, 11th edition. St. Louis: Mosby 148-168 pp.

6. National Committee for Clinical Laboratory Standards (NCCLS) (2004) Performance standards for antimicrobial susceptibility testing. Fourteenth Informational Supplement. Document M100-S14. Wayne, PA: NCCLS.

7. Mehrgan H, Rahbar M (2006) In vitro activity of imipenem, cefepime and ceftriaxone against common Gram-negative bacilli causing nosocomial infections. Iranian J Pharm Sci 2: 247 (P447).

8. Winokur PL, Canton R, Casellas JM, Legakis N (2001) Variations in the prevalence of strains expressing an extended-spectrum $\beta$-lactamase phenotype and characterization of isolates from Europe, the Americas, and the Western Pacific region. Clin Infect Dis 32: S94-S103.

9. Kader AA and Kumar A (2005) Prevalence and antimicrobial susceptibility of extended-spectrum $\beta$ lactamase-producing Escherichia coli and Klebsiella pneumoniae in a general hospital. Ann Saudi Med 25: 239242.

10. Daoud Z and Hakime N (2003) Prevalence and susceptibility patterns of extended-spectrum betalactamaseproducing Escherichia coli and Klebsiella pneumoniae in a general university hospital in Beirut, Lebanon. Rev Esp Quimioterap 16: 233-238.

11. Bouchillon SK, Johnson BM, Hoban DJ, Johnson JL, Dowzicky MJ, Wu DH, Visalli MA, Bradford PA (2004) Determining incidence of extended spectrum $\beta$-lactamase producing Enterobacteriaceae, vancomycin-resistant Enterococcus faecium and methicillin-resistant Staphylococcus aureus in 38 centres from 17 countries: the PEARLS study 2001-2002. Int J Antimicrob Agents 24: 119-124.

12. Bishara J, Livne G, Ashkenazi S, Levy I, Pitlik S, Ofir O, Lev B, Samra Z (2005) Antibacterial susceptibility of Extended-Spectrum Beta-Lactamase-Producing Klebsiella pneumoniae and Escherichia coli. Isr Med Assoc J 7: 298301.

13. Navon-Venezia S, Hammer-Munz O, Schwartz D, Turner D, Kuzmenko B, Carmeli Y (2003) Occurrence and phenotypic characteristics of extended-spectrum $\beta$-lactamases among members of the family Enterobacteriaceae at the Tel-Aviv medical center (Israel) and evaluation of diagnostic tests. $\mathbf{J}$ Clin Microbiol 41: 155-158.

14. Yaman A, Tasova Y, Kibar F, Inal AS, Saltoglu N, Buyukcelik O, Kurtaran B, Dundar IH (2004) Investigation of the antibiotic susceptibility patterns of pathogens causing nosocomial infections. Saudi Med J 25: 1403-1409.

15. Shehabi AA, Mahafzah A, Baadran I, Qadar FA, Dajani N (2000) High incidence of Klebsiella pneumoniae clinical 
isolates to extended-spectrum $\beta$-lactam drugs in intensive care units. Diagn Microbiol Infect Dis 36: 53-56.

16. Jabeen K, Zafar A, Hasan R (2005) Frequency and sensitivity pattern of Extended Spectrum Beta Lactamase producing isolates in a tertiary care hospital laboratory of Pakistan. J Pak Med Assoc 55: 436-439.

17. Mathur P, Kapil A, Das B, Dhawan B (2002) Prevalence of extended spectrum $\beta$-lactamase producing Gram-negative bacteria in a tertiary care hospital. Indian $\mathbf{J}$ Med Res 115: 153-157.

18. Giamarellou H (2005) Multidrug resistance in Gramnegative bacteria that produce extended-spectrum $\beta$ lactamases (ESBLs). Clin Microbiol Infect 11: 1-16.

19. Huber TW, Thomas JS (1994) Detection of resistance due to inducible $\beta$-lactamase in Enterobacter aerogenes and Enterobacter cloacae. J Clin Microbiol 32: 2481-2486.

20. Bell JM, Turnidge JD, Gales AC, Pfaller MA, Jones RN, the SENTRY APAC Study Group (2002) Prevalence of extended spectrum beta-lactamase (ESBL)-producing clinical isolates in the Asia-Pacific region and South Africa: regional results from SENTRY antimicrobial surveillance program (1998-99). Diagn Microbiol Infect Dis 42: 193-198.

21. Graffunder EM, Preston KE, Evans AM, Venezia A (2005) Risk factors associated with extended-spectrum $\beta$ lactamase-producing organisms at a tertiary care hospital. J Antimicro Chemother 56: 139-145.

22. Gupta A, Ampofo K, Rubenstein D, Saiman L (2003) Extended-spectrum $\beta$ lactamase-producing Klebsiella pneumoniae infections: a review of the literature. J Perinatol 23: 439-443.

23. Ozgunes I, Erben N, Kiremitci A, Kartal ED, Durmaz G, Colak H, Usluer G, Colak E. (2006) The prevalence of extended-spectrum beta lactamase-producing Escherichia coli and Klebsiella pneumoniae in clinical isolates and risk factors. Saudi Med J 27: 608-612.

24. Jones RN, Pfaller MA, MYSTIC Study Group (Europe) (2003) Antimicrobial activity against strains of Escherichia coli and Klebsiella spp. with resistance phenotypes consistent with an extended-spectrum $\beta$-lactamase in Europe. Clin Microbiol Infect 9: 708-712.

25. Pillary T, Pillary DG, Adhikari M, Sturm AW (1998) Piperacillin/tazobactam in the treatment of Klebsiella pneumoniae infections in neonates. AM J Perinatol 15: 4751.

\section{Corresponding author}

Dr. Hadi Mehrgan

Department of Pharmaceutics, School of Pharmacy, Shahid Beheshti University of Medical Sciences

P.O.Box 14155-6153

Vali-e-Asr Avenue, Niayesh Junction

Tehran, Iran

Tel: +98 21 88200068; Fax: +982188209620.

E-mail: hmehrgan75@yahoo.com

Conflict of interests: No confict of interests is declared. 